\title{
Is a Solitary Fibrous Tumor in the External Auditory Canal Benign?
}

\author{
Chi-Kyou Lee ${ }^{1}$ and Hyun Ju Lee ${ }^{2}$ \\ ${ }^{1}$ Departments of Otorhinolarygology-Head and Neck Surgery, ${ }^{2}$ Pathology, Soonchunhyang University School of Medicine, \\ Cheonan, Korea
}

\section{Received January 20, 2016 \\ Revised March 28, 2016 \\ Accepted March 31, 2016}

Address for correspondence

Chi-Kyou Lee, MD, PhD

Department of Otorhinolarygology-

Head and Neck Surgery,

Soonchunhyang University

School of Medicine,

31 Suncheonhyang 6-gil,

Dongnam-gu, Cheonan 31151, Korea

Tel $+82-41-570-2353$

Fax +82-41-579-9022

E-mail_drqlee@hanmail.net
A solitary fibrous tumor (SFT) is an extremely rare, distinct otological soft-tissue tumor. Only two such tumors in the external auditory canal have been reported. A SFT related to hemangiopericytomas (HPC), which commonly arise in the central nervous system. HPCs act malignant in many cases, while SFTs at other sites are mainly benign. A 25-year-old female presented with highly vascular tumor at the right external auditory meatus and bleeding from the mass when a crust was removed from its surface. After excisional biopsy followed by pre-operative embolization, this tumor confirmed with SFT. The recurrence rate of SFT is very low after complete resection, with a slightly increased risk of recurrence with extrathoracic tumors. We describe the third case of SFT in the external auditory canal and review the literature.

J Audiol Otol 2016;20(2):120-122

KEY WORDS: External auditory canal · Solitary fibrous tumor · Hemangiopericytoma.

\section{Introduction}

Various tumors can arise in the external auditory canal (EAC). A solitary fibrous tumor (SFT) is a distinct soft-tissue tumor related to hemangiopericytomas (HPCs), which commonly arise in the central nervous system and pleura. Extrapleural SFTs have been reported in the liver, nasopharynx, nasal cavity, paranasal sinuses, oral cavity, thyroid, sublingual, and parotid glands [1]. Their classification is based mainly on histopathology, especially immunochemical staining for CD34 antibody. SFTs are extremely rare in otology. Two cases reported by Izumaru, et al. [1] and Rezk, et al. [2]. Here, we describe the third case of SFT in the EAC and review the literature.

\section{Case Report}

A 25-year-old female presented with progressive swelling

This is an Open Access article distributed under the terms of the Creative Commons Attribution Non-Commercial License (http://creativecommons.org/licenses/by-nc/3.0/) which permits unrestricted non-commercial use, distribution, and reproduction in any medium, provided the original work is properly cited. at the right external auditory meatus and bleeding from the mass when a crust was removed from its surface. Examination revealed a round ulcerated, skin-covered mass located on the posterior-superior wall of the entrance of the EAC, which partially obstructed the canal (Fig. 1A). The medial EAC, eardrum and an audiogram were normal. Contrast-enhanced computed tomography (CT) showed immensely enhanced round mass with no invasion of adjacent structures and normal middle ear cavity (Fig. 1B). These findings strongly suggested that the tumor originated from vascular tissue. Four-vessel angiography showed that it was fed by the posterior auricular artery, which was embolized successfully (Fig. 1C, D). Under general anesthesia, the tumor was excised under an operating microscope; there were no adhesions to the surrounding tissues. The defect in the EAC was covered with a full-thickness retroauricular skin graft. The surgical specimen measured $2.0 \times 1.8 \times 1.5 \mathrm{~cm}$ with attached skin $(1.8 \times 1.0 \mathrm{~cm})$. On cut section, the tumor was a well-demarcated, non-encapsulated, solid, gray-white nodule in the dermis (Fig. 2A). There was no necrosis or hemorrhage. Histologically, the mass comprised predominantly spindle cells of moderate cellularity in a patternless architecture of hypo- and hypercellular areas separated by thick, hyalinized collagen with 

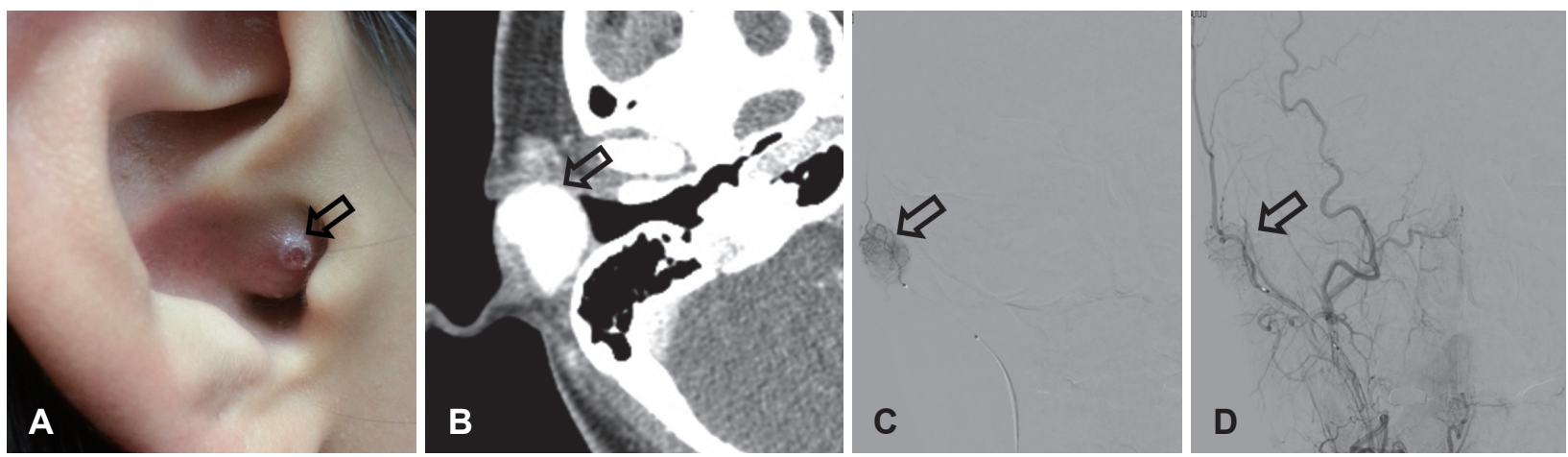

Fig. 1. A: A small, ulcerated, skin-covered mass (arrow) on the posterosuperior wall partially blocking the entrance of the external auditory canal. B: Contrast-enhanced CT showed a round hyperattenuated mass (arrow) with no clear invasion of adjacent structures. C, D: Four-vessel angiography was used to find and embolize the feeding artery, the posterior auricular artery. C: Round dark mass (arrow) before shutting off. D: Faint mass shadow (arrow) after shutting off.
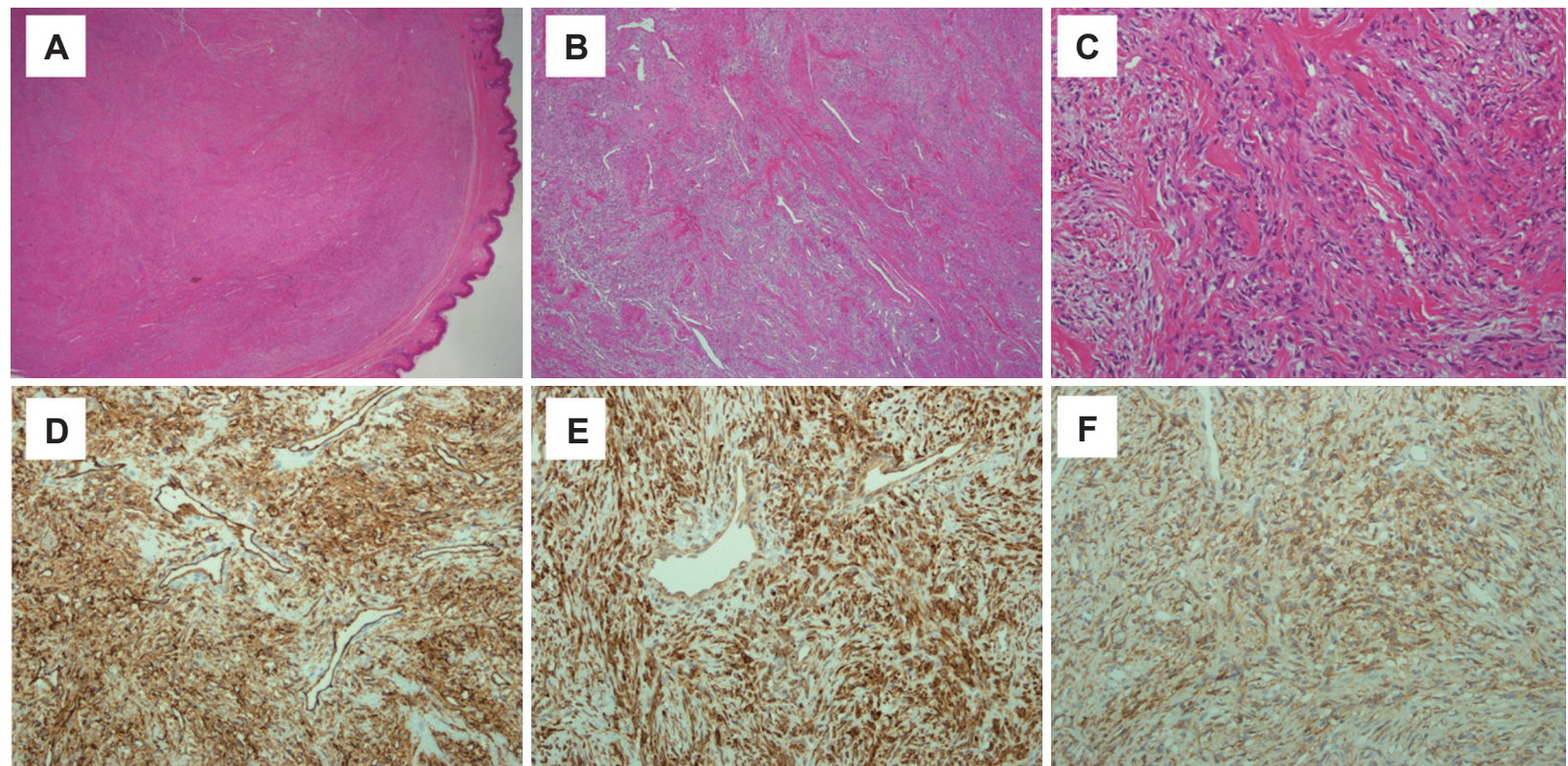

Fig. 2. Histological and immunohistochemical findings of the solitary fibrous tumor. Well-circumscribed, non-encapsulated spindle-cell tumor located in the dermis (A) (H\&E, $\times 10)$. The tumor had a patternless architecture of hypo- and hypercellular areas separated by thick, hyalinized collagen with cracking artifact and staghorn vessels (B) (H\&E, $\times 40)$. Fibroblast-like cells with bland nuclear features, mixed with thick bands of collagen (C) (H\&E, $\times 200)$. The spindle cells are positive for CD34 (D) ( $\times 200)$, and partially positive for bcl2 $(\mathrm{E})(\times 200)$ and $\operatorname{CD} 99(\mathrm{~F})(\times 200)$.

occasional vascular clefts (Fig. 2B) with focal myxoid stroma. The tumor cells had minimal nuclear pleomorphism (Fig. 2C). Roughly two mitotic figures were found per ten highpower fields, but no atypical mitotic figures were identified. Immunohistochemically, tumor cells showed a positive reaction for CD34, and partially positive for bcl2 (Fig. 2E) $(\times 200)$ and CD99 (Fig. 2F) (×200). (Fig 2D, F) But they did show a negative reaction for cytokeratin, epithelial membrane antigen, S-100 protein, smooth muscle actin, CD68 and CD31. The Ki-67 proliferative index was $2 \%$. Based on these findings, it was diagnosed as a SFT.

\section{Discussion}

SFTs occur equally in males and females at a median age of 45-50 years and are less common in infants and children [2]. Soft-tissue SFTs represent 1-2\% of all soft-tissue tumors [3]. Klemperer and Coleman [4] first described a SFT in 1992, as a pleural neoplasm. The pathogenesis of SFT is controversial; a mesenchymal cell origin is currently favored over a mesothelial origin [5]. Despite their characteristic histological and immunohistochemical features, many clinicians are still unfamiliar with SFTs of the head and neck, which are frequently misdiagnosed as other neoplasms that are more common in the head and neck, such as angiofibroma. Our 
case had an abundant blood supply, so we had to rule out other vascular tumors. An HPC is a vascular tumor of the head and neck, first described in 1942 as a distinct neoplasm of pericytic origin that tends to be malignant [6], but over $15 \%$ of all soft-tissue tumors have the staghorn-branching vascular pattern representative of HPC focally [7]. Currently, softtissue pathologists prefer the term SFT to describe a rare, heterogeneous group of benign or malignant neoplasms along a morphological spectrum: pleural SFT, soft-tissue SFT, and meningeal HPC [3]. The fibrous form is distinguished by hyalinized, thick-walled vessels with open lumens and strong CD34 reactivity. The cellular form, represented by typical HPC, features branching, thin-walled vessels and focal or absent CD34 reactivity [7].

On CT, a SFT can be hypo- or hyperattenuated relative to muscle, depending on collagen content [8]. Heterogeneous enhancement is seen in $100 \%$ of malignant and $60 \%$ of benign SFTs [9]. If the CT contrast enhancement is high, the tumor might be malignant and serious bleeding can follow surgical excision [1]. Therefore, it is preferable to embolize the feeding vessel preoperatively, enabling unimpeded tumor removal with an excellent surgical view, as in our case.

Most SPTs are slow-growing and benign, although malignant pleural and extrapleural tumors have been documented $[1,10]$. Consequently, such tumors should be resected as completely as possible. The recurrence rate is very low after complete resection, with a slightly increased risk of recurrence with extrathoracic tumors [2]. Malignant tumors show high cellularity, increased mitotic activity ( $>4$ per 10 high-power fields), pleomorphism, extensive hemorrhage, and necrosis; tumors larger than $10 \mathrm{~cm}$ should be monitored closely [2]. Using these criteria, our case was likely benign. However, this is inferred mainly from data on pleural SFTs or sites other than the ear canal. As there are no long-term follow-up reports on SFT in the auditory canal, we counseled the patient carefully about the malignant potential. She is under close followup without recurrence over two years.

\section{Conflicts of interest}

The authors have no financial conflicts of interest.

\section{REFERENCES}

1) Izumaru S, Yoshida Y, Nakashima T. A solitary fibrous tumor in the external auditory meatus. Auris Nasus Larynx 2004;31:65-7.

2) Rezk S, Yousef M, Zamansky M, Khan A. Solitary fibrous tumor of the auditory canal. Arch Pathol Lab Med 2004;128:e169-71.

3) Penel N, Amela EY, Decanter G, Robin YM, Marec-Berard P. Solitary fibrous tumors and so-called hemangiopericytoma. Sarcoma 2012;2012:690251.

4) Klemperer P, Coleman BR. Primary neoplasms of the pleura. A report of five cases. Am J Ind Med 1992;22:1-31.

5) England DM, Hochholzer L, McCarthy MJ. Localized benign and malignant fibrous tumors of the pleura. A clinicopathologic review of 223 cases. Am J Surg Pathol 1989;13:640-58.

6) Stout AP, Murray MR. Hemangiopericytoma: a vascular tumor featuring zimmermann's pericytes. Ann Surg 1942;116:26-33.

7) Gengler C, Guillou L. Solitary fibrous tumour and haemangiopericytoma: evolution of a concept. Histopathology 2006;48:63-74.

8) Ginat DT, Bokhari A, Bhatt S, Dogra V. Imaging features of solitary fibrous tumors. AJR Am J Roentgenol 2011;196:487-95.

9) Rosado-de-Christenson ML, Abbott GF, McAdams HP, Franks TJ, Galvin JR. From the archives of the AFIP: Localized fibrous tumor of the pleura. Radiographics 2003;23:759-83.

10) Ogawa I, Sato S, Kudo Y, Miyauchi M, Sugiyama M, Suei Y, et al. Solitary fibrous tumor with malignant potential arising in sublingual gland. Pathol Int 2003;53:40-5. 